REVIEW

\title{
Application of toxicological risk assessment principles to the chemical constituents of cigarette smoke
}

\section{J Fowles, E Dybing}

See end of article for authors' affiliations

.....................

Correspondence to: Jeff R Fowles, Institute of Environmental Science and Research, Ltd, PO Box 50-348, Porirua, New Zealand; jeff.fowles@ esr.cri.nz

Received 10 February 2003 Accepted

9 September 2003
Objective: To provide a hazard prioritisation for reported chemical constituents of cigarette smoke using toxicological risk assessment principles and assumptions. The purpose is to inform prevention efforts using harm reduction.

Data sources: International Agency for Research on Cancer Monographs; California and US Environmental Protection Agency cancer potency factors (CPFs) and reference exposure levels; scientific journals and government reports from the USA, Canada, and New Zealand.

Study selection: This was an inclusive review of studies reporting yields of cigarette smoke constituents using standard ISO methods.

Data extraction: Where possible, the midpoint of reported ranges of yields was used.

Data synthesis: Data on 158 compounds in cigarette smoke were found. Of these, 45 were known or suspected human carcinogens. Cancer potency factors were available for 40 of these compounds and reference exposure levels (RELs) for non-cancer effects were found for 17. A cancer risk index (CRI) was calculated by multiplying yield levels with CPFs. A non-cancer risk index (NCRI) was calculated by dividing yield levels with RELs. Gas phase constituents dominate both CRI and NCRI for cigarette smoke. The contribution of 1,3-butadiene (BDE) to CRI was more than twice that of the next highest contributing carcinogen (acrylonitrile) using potencies from the State of California EPA. Using those potencies from the USEPA, BDE ranked third behind arsenic and acetaldehyde. A comparison of CRI estimates with estimates of smoking related cancer deaths in the USA showed that the CRI underestimates the observed cancer rates by about fivefold using ISO yields in the exposure estimate.

Conclusions: The application of toxicological risk assessment methods to cigarette smoke provides a plausible and objective framework for the prioritisation of carcinogens and other toxicant hazards in cigarette smoke. However, this framework does not enable the prediction of actual cancer risk for a number of reasons that are discussed. Further, the lack of toxicology data on cardiovascular end points for specific chemicals makes the use of this framework less useful for cardiovascular toxicity. The bases for these priorities need to be constantly re-evaluated as new toxicology information emerges.
C igarettes are probably the single most significant source of toxic chemical exposure and chemically mediated illness in humans. The World Health Organization forecasts cigarettes will kill nearly 10 million people per year globally by the year $2020 .{ }^{1}$ However, despite the public health significance of these toxicant exposures, comparatively little effort has been spent to assess and quantify the health risks associated with the individual chemicals in cigarette smoke, and almost nothing has been done in terms of product regulation to reduce human exposure to these constituents.

All of the necessary components of the risk assessment framework-hazard identification, hazard characterisation, exposure assessment, and risk characterisation-can be used to address cigarette smoke constituents. While there remain legitimate concerns about the relation between smoking machine "yield" and actual human uptake in absolute terms, the relative concentrations of toxicants within a set of tobacco products can still be compared.

Newly emerging tobacco products, notably products that claim to heat, rather than burn, tobacco come with claims of reduced toxicant yields and reduced harmfulness as they are test marketed internationally. ${ }^{2}$ At the same time, governments are starting to require smoke constituents to be analysed and reported. ${ }^{3}$ In light of these developments, it is important to develop toxicological risk assessment methods that can help evaluate the public health significance of the product modifications and any analytical results that are generated.
It is unlikely that a "safe cigarette" could ever be developed, as combustion products in smoke are inherently potentially harmful. Nevertheless, it may be possible to reduce some of the toxic potency of cigarettes if the most significant causative agents of disease can be identified and reduced or eliminated. However, it is important that such a strategy does not give the public the false impression that cigarette smoking has become a safe practice. Such a perception could counteract any public health gains made by the reduction of the toxicity of tobacco smoke.

Ideally, policies to do with prevention of smoking would concentrate on preventing people from starting to smoke or assisting them to stop smoking entirely. However, such policies have been only partially successful, with the worldwide number of smoking related deaths at around 4 million annually and climbing. ${ }^{1}$

This paper presents a review of the chemical constituents found in cigarette smoke, and provides an analysis of the

\footnotetext{
Abbreviations: BDE, 1,3-butadiene; Cal/EPA, California

Environmental Protection Agency; CRI, cancer risk indices; CPF, cancer potency factor; IARC, International Agency for Research on Cancer; ISO, International Organization for Standardization; NAB, Nnitrosoanabasine, NAT, N-nitrosoanatabine; NCRI, non-cancer risk index; REL, reference exposure level; USEPA, US Environmental Protection Agency; TCDD, 2,3,7,8-tetrachlorodibenzo-p-dioxin
} 
relative contribution each constituent would be expected to make to health risks, based on published hazard and constituent content values.

\section{METHODS}

\section{Constituents of smoke}

A comprehensive search, using the internet and bibliographic databases, was carried out for published papers and reports dealing with the constituents of tobacco smoke and assessments of their toxic hazards.

Reported levels of chemicals in cigarette smoke were taken from all available published sources, without discrimination as to publishing author affiliation. No single information source of chemicals in cigarette smoke was found, and so it was necessary to piece together information on chemicals and yields from a variety of published sources. Although many more chemicals than what are presented in this paper exist in cigarette smoke, the chemicals listed are those that have been quantified and reported with toxicological potency estimates in the literature.

The identified chemicals were examined for the evidence of their influence on the toxicity of cigarette smoke. A process of prioritisation (ranking) of these chemical hazards was applied. This process involved ranking the identified chemicals in terms of their so called comparative risk indices. The comparative risk ranking was based on published analytical results for mainstream cigarette smoke combined with published toxicological potency information for cancer and non-cancer health effects.

We have assumed, for the sake of the current analysis, that the most significant toxicants in cigarette smoke are those that have been identified and studied toxicologically. For yield estimates per cigarette, reports using standard smoking machine conditions and International Organization for Standardization (ISO) methods were taken in preference to those focusing on "intense" smoking conditions simply in order to promote consistency when comparing relative yields of specific chemicals between studies. In the exposure estimates, no additional contribution to mainstream smoke exposure from sidestream smoke was included. These assumptions are likely to result in an underestimate of the absolute level of exposure and associated risk to the smoker from each constituent.

\section{Cancer potency factors}

Published cancer potency factors (CPFs) from the state of California's Proposition 65 list of chemicals "Known to the state to cause cancer" were used to define the dose-response relation for suspected or known carcinogens in cigarette smoke. For comparison, we included the US Environmental Protection Agency (EPA) inhalation cancer potency factors, although there were only 17 of these available with relevance to tobacco smoke, compared with California's 40. CPFs were not available for all carcinogenic compounds. Also, CPFs were only used here for compounds with known or potential genotoxic activity, which effectively excludes any possible contribution from chlorinated dioxins and furans. Compounds with unknown tumour initiating mechanisms (for example, acetamide ${ }^{4}$ ) were included in the overall cancer hazard ranking. Cancer potency estimates were usually derived from one or more animal studies using a linearised multistage model with 95\% confidence intervals on the slope term. We used the "unit risk" form of these CPFs as $(\mu \mathrm{g} /$ $\left.\mathrm{m}^{3}\right)^{-1}$ to allow for inhalation exposures. These CPFs have been developed and approved by risk assessment experts at the California Environmental Protection Agency (Cal/EPA) as part of the state's Proposition 65 program. ${ }^{5}$ Many of these factors are the same as those found in the US Environmental
Protection Agency (USEPA) Integrated Risk Information System. ${ }^{6}$

A similar approach has been reported by the state of Massachusetts, ${ }^{7}$ and for the New Zealand Ministry of Health. ${ }^{8}$

\section{Cancer risk indices}

The cancer risk indices (CRI) for mainstream smoke were calculated on a per cigarette per day basis by multiplying the yield per cigarette $(\mu \mathrm{g})$ with the published cancer potency factor, assuming complete absorption (in the case of smokers) of the chemicals in the reported yield. It was also assumed in the calculations that exposure would take place for an average of 60 years out of a 75 year lifespan, for an average person.

As an example of the CRI calculations, the level for BDE (35.5 $\mu \mathrm{g} /$ cigarette) in mainstream smoke (see table 1 on TC online at http://www.tobaccocontrol.com/supplemental) was divided by a default breathing rate of $20 \mathrm{~m}^{3} /$ day, and then multiplied by the published cancer potency factor of $1.7 \mathrm{E}-04\left(\mu \mathrm{g} / \mathrm{m}^{3}\right)^{-1}$ to give a comparative CRI value of $3.0 \times 10^{-4}$ for a person smoking for 60 years: cancer risk index $=\left[35.5 \mu \mathrm{g} / \mathrm{cig} / 20 \mathrm{~m}^{3} /\right.$ day $] \times 1.7 \mathrm{E}-04\left(\mu \mathrm{g} / \mathrm{m}^{3}\right)^{-1}=$ 0.00030 per cigarette per day. This then is modified depending on the number of years out of a typical lifespan one selects as appropriate for a given smoker.

\section{Dioxins}

Dioxins, as they relate to 2,3,7,8-tetrachlorodibenzo-p-dioxin (TCDD) equivalents, are in the International Agency for Research on Cancer's (IARC) group 1 as known human carcinogens. However, the toxicity of dioxin is receptor mediated, it is not genotoxic, and there is likely a threshold below which individual exposures would not be expected to cause elevated risk of cancer. Therefore, while the TCDD cancer unit risk is shown in table 1, it is not factored into the overall cancer hazard scheme. Acceptable/tolerable daily intakes (reference levels) have also been developed for TCDD. ${ }^{9}$ For these reasons, dioxins were not included in the quantitative CRI calculations despite their reported occurrence in cigarette smoke. ${ }^{10}$

\section{Non-cancer risk indices}

For the prioritisation of constituents with known non-cancer health effects, a non-cancer risk index (NCRI) was derived based on target organ toxicity. NCRIs for cardiovascular and respiratory health effects from mainstream smoke were generated. The NCRI for mainstream smoke was calculated using publicly available reference exposure levels (RELs) with respective target organs listed by the USEPA or Cal/EPA. ${ }^{5}{ }^{10}$ These are also listed in table 1. A standard $20 \mathrm{~m}^{3} /$ day breathing rate default value was used for estimating exposures and converting RELs into units of $\mu \mathrm{g} /$ person/day in the case of smokers.

\section{Reference exposure levels}

REL from the USEPA or Cal/EPA for chronic, organ specific effects were used. ${ }^{511}$ These RELs can be viewed as practical threshold levels below which one would not expect to measure any adverse effects with chronic exposure. The probability of disease is a function of the degree of exposure above the threshold value, but any exposure above the threshold may represent some level of increased health risk above background. The RELs contain margins of safety ranging from a factor of 1 to a factor of 1000, depending on the data used as the basis for the toxicological effect. These uncertainty factors are used to provide a margin of safety to account for variability in human response or uncertainties in extrapolation from controlled experimental animal studies to what is actually experienced by the general public. These 
RELs have been used to calculate a hazard index for each chemical, relative to its measured concentrations in mainstream smoke.

\section{Overall non-cancer risk indices}

The underlying assumption behind the NCRI approach is that the toxicity of each individual chemical in a given mixture is additive with other chemicals that affect the same target tissue or organ system. All NCRIs assume there is a threshold exposure below which adverse effects are not expected to occur. The necessary components of an NCRI calculation are: a measured or estimated exposure to the chemical, and a health hazard benchmark concentration or reference dose for comparison (the REL). The REL is taken from the most sensitive effect reported in the toxicological or epidemiological literature combined with an appropriate uncertainty factor. The value used for comparison carries a specification as to the target organ or system for a given toxic effect. A chemical often has more than one target organ, in which case, to be health protective, the REL for the most sensitive effect is also applied to the other target organs.

The overall NCRI was calculated as follows: Overall NCRI $=\mathrm{E}_{1} / \mathrm{REL}_{1}+\mathrm{E}_{2} / \mathrm{REL}_{2}+\ldots \mathrm{E}_{\mathrm{n}} / \mathrm{REL}_{\mathrm{n}}$ where $\mathrm{E}=\mathrm{a}$ measured or estimated exposure to a chemical, and REL is the chemical's reference exposure level for a given target organ effect. An NCRI $>1$ implies that the threshold for toxic effects on the target organ has been exceeded. The NCRI calculations for mainstream smoke are based on the reported yields of a single cigarette. Clearly, most smokers consume more than a single cigarette per day. However, the purpose in this analysis is to provide a comparative ranking of exposure to tobacco smoke constituents, rather than an assessment of actual risk. Therefore, we did not attempt to take into account the number of cigarettes smoked, or how they might be smoked, and provide an absolute measure of risk for a given smoker. For the purposes of this report, the NCRI values provide a basis for prioritisation of non-cancer adverse health effect concerns about known chemicals in cigarette smoke.

\section{RESULTS}

\section{Chemicals found in cigarette smoke}

A list of chemicals in mainstream and sidestream smoke, IARC classification, cancer potencies, and non-cancer reference exposure levels is shown in table 1 . In all, 158 chemical constituents of smoke were identified where this information was found. Fifty two of these compounds are known or suspected carcinogens according to IARC. One hundred and nineteen of the compounds had quantified concentrations (yields) for mainstream smoke reported in the open literature or by the British Columbia Ministry of Health ${ }^{3}$ (http:// www.cctc.ca). Of these, 45 are known or suspected carcinogens under the IARC classification scheme ${ }^{12}$ : group 1 (known human carcinogens) or $2 \mathrm{~A}$ (probable human carcinogens) or group 2B (possible human carcinogens). Group 3 chemicals (not classifiable) are shown in table 1 , but did not factor into the CRI calculations. CPFs expressed as $\left(\mu \mathrm{g} / \mathrm{m}^{3}\right)^{-1}$ were available for 40 of the 45 chemicals. Of those known or suspected carcinogens not having an available CPF, NNK appeared to be the most prominent in terms of exposure and suspected potency. Since a CPF did not exist for NNK, the published CPF for NNN was used as a surrogate for NNK.

An additional 17 chemicals had non-cancer reference exposure levels for threshold type effects. ${ }^{11}$

\section{Ranking individual cancer hazards}

Table 2 lists the results of CRI calculations for mainstream smoke. The constituent showing the highest relative contribution to CRI is 1,3-butadiene (BDE) using Cal/EPA CPFs
Table 2 Summary table of cancer risk indices for individual chemical constituents of cigarette smoke based on exposure to a single cigarette per day

\begin{tabular}{|c|c|c|}
\hline $\begin{array}{l}\text { Toxicant } \\
\text { category* }\end{array}$ & Mainstream smoke constituent & $\begin{array}{l}\text { CRI per cigarette/ } \\
\text { day }\end{array}$ \\
\hline 1 & 1,3-Butadiene & $3.02 \mathrm{E}-04$ \\
\hline 1 & Acrylonitrile & $1.29 \mathrm{E}-04$ \\
\hline 2 & Arsenic & $1.16 \mathrm{E}-04$ \\
\hline 1 & Acetaldehyde & $9.18 \mathrm{E}-05$ \\
\hline 1 & Benzene & $6.71 E-05$ \\
\hline 1 & Acetamide & 4.70E-05 \\
\hline 3 & N-Nitrosonornicotine (NNN) & $3.80 \mathrm{E}-05$ \\
\hline 3 & N-Nitrosopyrrolidine (NP) & 3.39E-05 \\
\hline 2 & Chromium (hexavalent) & $3.15 \mathrm{E}-05$ \\
\hline 2 & Cadmium & $2.16 \mathrm{E}-05$ \\
\hline 1 & Formaldehyde & $9.90 E-06$ \\
\hline 4 & Hydrazine & $8.33 \mathrm{E}-06$ \\
\hline 3 & $\begin{array}{l}\text { 4-N-Nitrosomethylamino)-1-(3- } \\
\text { pyridyl)-1-butanone (NNK) }\end{array}$ & 7.80E-06† \\
\hline 3 & N-Nitrosodimethylamine (NDMA) & $5.58 \mathrm{E}-06$ \\
\hline 4 & DDT & 4.61E-06 \\
\hline 3 & N-Nitrosodiethylamine (NDEA) & 4.27E-06 \\
\hline 5 & Benzo(a)pyrene & $1.93 \mathrm{E}-06$ \\
\hline 3 & N-Nitroso-N-dibutylamine & $1.89 \mathrm{E}-06$ \\
\hline 3 & N-Nitrosoethyl-methylamine & $1.89 \mathrm{E}-06$ \\
\hline 5 & Dibenzo(a,i)pyrene & $1.38 \mathrm{E}-06$ \\
\hline 3 & N-Nitrosodiethanolamine & $1.20 \mathrm{E}-06$ \\
\hline 3 & N Nitrosopiperidine & $6.04 \mathrm{E}-07$ \\
\hline 1 & Urethane & 4.14E-07 \\
\hline 4 & 4-Aminobiphenyl & $3.60 \mathrm{E}-07$ \\
\hline 5 & Benzo(j)fluoranthene & $3.30 \mathrm{E}-07$ \\
\hline 5 & Benz(a)anthracene & $2.48 \mathrm{E}-07$ \\
\hline 5 & Dibenz $(a, h)$ anthracene & $2.40 \mathrm{E}-07$ \\
\hline 5 & 2-Aminonaphthalene & $1.80 \mathrm{E}-07$ \\
\hline 5 & Benzo(b)fluoranthene & $1.65 \mathrm{E}-07$ \\
\hline 2 & Nickel & $1.43 \mathrm{E}-07$ \\
\hline 5 & Indeno(1,2,3-c,d)pyrene & $6.60 \mathrm{E}-08$ \\
\hline 3 & $\mathrm{~N}$-Nitroso-N-propylamine & $5.00 \mathrm{E}-08$ \\
\hline 5 & Benz $(k)$ fluoranthene & $4.95 \mathrm{E}-08$ \\
\hline 5 & 7H-Dibenzo(c,g)-carbazole & $3.85 \mathrm{E}-08$ \\
\hline 1 & Vinyl chloride & $3.35 \mathrm{E}-08$ \\
\hline 5 & 5-Methylchrysene & 3.30E-08 \\
\hline 2 & Beryllium & $3.00 \mathrm{E}-08$ \\
\hline 5 & Chrysene & $2.75 \mathrm{E}-08$ \\
\hline 5 & Dibenz(a,j)acridine & $1.49 \mathrm{E}-08$ \\
\hline 2 & Lead & $7.68 \mathrm{E}-09$ \\
\hline 5 & Dibenz(a,h)acridine & $5.50 \mathrm{E}-10$ \\
\hline
\end{tabular}

${ }^{*} 1$, aldehydes and small organics from combustion; 2, metals; 3, nitrosamines; 4, miscellaneous compounds; and 5, polycyclic aromatics (PAHs).

†No cancer unit risk value was available for NNK. The unit risk from NNN was used as a surrogate for this tobacco specific nitrosamine.

and arsenic using USEPA CPFs. Using the Cal/EPA scheme, which contained 40 potencies as compared with 17 from the USEPA, the contribution to the overall cancer risk from BDE in mainstream smoke was more than twofold greater than that of the next highest carcinogen.

CRIs, calculated using Cal/EPA or USEPA potency factors, were summed for various classes of compounds, and for the Cal/EPA are as follows (in decreasing order): aldehydes and other small volatile organic combustion products combined for $70 \%$ (including benzene), metals (cadmium, arsenic, beryllium and lead) $18 \%$, nitrosamines $10 \%$, and PAHs $1 \%$. The remaining constituents combined for less than $2 \%$ of the CRIs (fig l).

The chemical with by far the greatest impact on the CRI calculation if Cal/EPA potencies are used, is BDE, with more than twice the cancer risk contribution of the next highest chemical (acrylonitrile). Using the USEPA potencies, BDE still contributes strongly, but is third behind arsenic and acetaldehyde. Using the USEPA cancer potencies (fig 2), the contributions to overall CRI are as follows: aldehydes and other small organic combustion products combined for 54\% (including benzene), metals (cadmium, arsenic, chromium, 


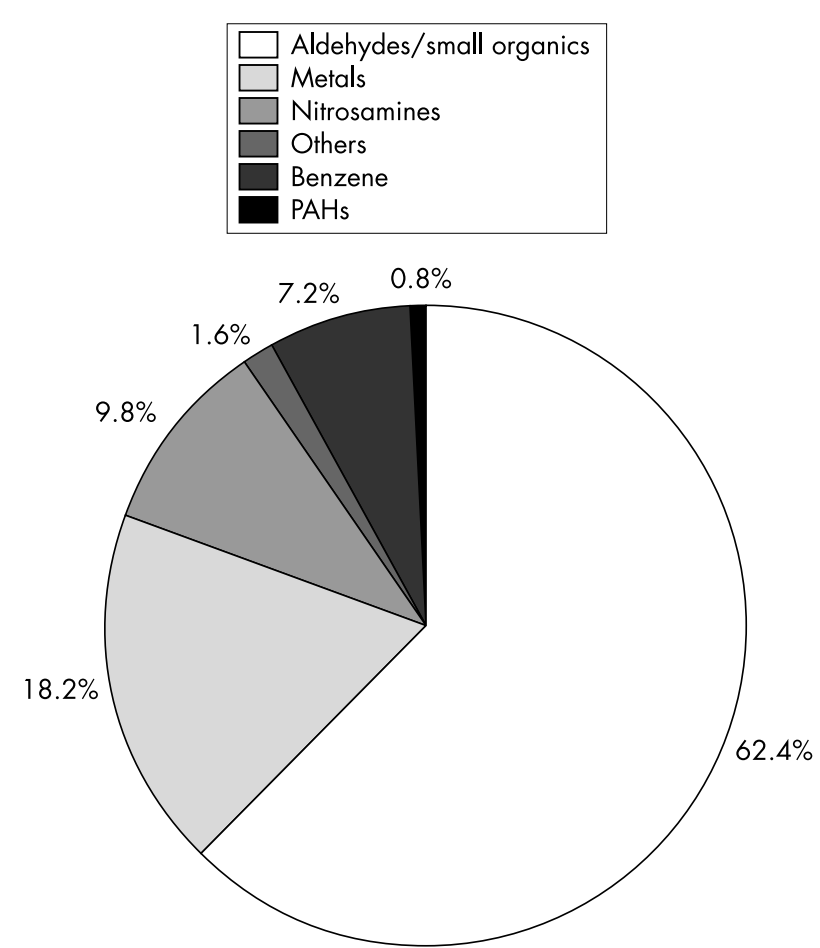

Figure 1 Contribution to cancer risk index of cigarette smoke by chemical class (Cal/EPA).

nickel, beryllium and lead) 34\%, nitrosamines $4.4 \%$, and others $3 \%$. The USEPA have no potency estimates for PAHs. The main contributors to the overall CRI from cigarette smoke, using either Cal/EPA or USEPA potencies, are therefore aldehydes and small organic compounds resulting from combustion. While the contribution from BDE dominates the CRI calculations, the relative contributions to CRI

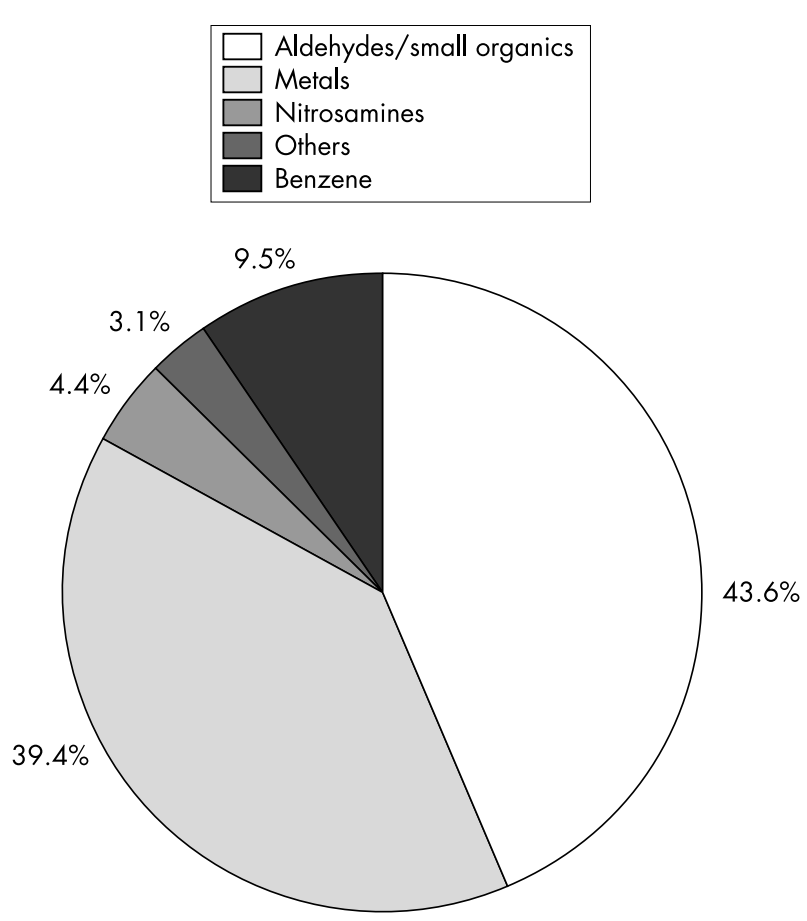

Figure 2 Contribution to cancer risk index of cigarette smoke by chemical class (USEPA). for the different chemical classes remains in the same ranked order even if BDE is excluded from the analysis.

\section{Comparing cancer potency factors with observed cancer death rates}

The focus of this paper is the ranking of hazards in the cigarette smoke stream. It is instructive however, to see how the sum of these factors relates to what is observed in smoking-related cancer statistics. A sum of the individual CRIs from table 2, assuming a person smoking chronically for 60 years out of a 75 year lifespan, gives a crude cancer risk estimate of $8.0 \times 10^{-4}$ per cigarette per day. This translates into about $1.6 \times 10^{-2}$ for a 20 cigarette pack per day. This result can be compared with reports of observed cancer incidence due to smoking.

For example, the expected male and female deaths from cancer in the USA in 1995 totalled 547 000, of which 168057 (or $30.7 \%$ ) are thought to have been due to cigarette smoking. ${ }^{21}$ If one assumes that the US smoker population consists of about $25 \%$ of the total population of approximately 263909000 (US Census of Resident Population, December 1995) this means there are 65977250 smokers dying annually from cancer in the USA. A lifetime cancer risk estimate for smokers would therefore be: [168 057 (cancer death from smoking)/65 977250 (number of smokers at 25\% prevalence) $] \times 70$ years $=0.178$ (or one in 5.6 chance of a smoker dying from smoking-related cancer).

Compared with cancers not attributable to smoking: [[547 $000-168057$ (cancer deaths not attributable to smoking)]/263 909000 (total resident population including smokers) $] \times 70$ years $=0.0986$ (or one in 10.1 chance of dying from a non-smoking related cancer).

Note-sidestream smoke cancers among non-smokers are not factored in, so this is an overestimate of the true cancer rates not attributable to cigarettes.

The excess cancer risk from smoking is therefore estimated at: $0.178-0.0986=0.079$ or $7.9 \times 10^{-2}$.

This value, from observed mortality statistics, is 4.9 times greater than that predicted by summing individual contributions to cancer risk of chemicals in smoke using CRIs. It is evident, therefore, that the crude approach of combining potencies with ISO smoking machine yields underestimates the actual cancer burden.

\section{Non-cancer effects}

The NCRIs rely on available RELs, but there were comparatively few RELs for non-cancer effects developed by the USEPA or Cal/EPA. In contrast to the cancer potencies, the non-cancer reference doses and reference exposure levels from the two agencies are very similar, differing only for benzene $\left(60 \mu \mathrm{g} / \mathrm{m}^{3} v 30 \mu \mathrm{g} / \mathrm{m}^{3}\right.$ ), and arsenic (USEPA has no value currently). Table 1 provides RELs for only 28 out of 158 chemicals in smoke $(18 \%)$. Some prominent chemicals are absent from the list of chemicals contributing to these risk areas, including nicotine's potential influence on cardiovascular disease and reproductive toxicity. The biological bases for these effects from nicotine have been described, ${ }^{22}$ but a practical threshold value is not available. The true magnitude of non-cancer risks are also underestimated here, due to gaps in dose-response information for chemicals (for example, nicotine) and corresponding definitive threshold values from authoritative sources.

Table 3 shows that the greatest contributors to respiratory irritation are aldehydes and small organic compounds resulting from combustion of organic material. Acrolein and acetaldehyde greatly outweigh the remaining compounds in regard to the contribution to respiratory irritation. The respiratory irritant effects of the compounds in table 3 are well studied and have a considerable amount of human 
Table 3 Summary table of non-cancer risk indices for individual chemical constituents of mainstream cigarette smoke based on a single cigarette per day

\begin{tabular}{lll}
\hline Non-cancer effects & Smoke constituent & NCRI \\
\hline Respiratory effects & Acrolein & $172^{*}$ \\
& Acetaldehyde & 3.78 \\
& Formaldehyde & 0.83 \\
& Cadmium & 0.52 \\
& Chromium (hexavalent) & 0.26 \\
& Acrylonitrile & 0.22 \\
& Nickel & 0.011 \\
Cardiovascular effects & Ammonia & 0.006 \\
& Hydrogen cyanide & 1.97 \\
& Arsenic & 1.17 \\
& m-+p-Cresol & 0.18 \\
& O-Cresol & 0.071 \\
& Carbon monoxide & 0.068 \\
& Benzene & 0.039 \\
& Phenol & 0.0022 \\
\hline
\end{tabular}

*Reference exposure levels ${ }^{6}{ }^{11}$ are intended to protect sensitive individuals against chronic effects over a chronic period of continuous exposure. The NCRI is equal to reported concentrations divided by the REL, assuming an average $20 \mathrm{~m}^{3} /$ day breathing rate. Bold numbers indicate an NCRI

$>1.0$ which signals that the threshold for adverse effects could be reached for some people from smoking a single cigarette per day.

data available. In contrast, the contributors to cardiovascular toxicity, with the exception of carbon monoxide, are less well studied and the mechanisms involved may not be additive. Carbon monoxide is widely thought to be the major contributor to cardiovascular disease from smoking, yet the indices in this analysis show that exposure to hydrogen cyanide, arsenic, and cresols may actually pose a greater cardiovascular risk. As shown in the bold type values in table 3, the individual risk indices of hydrogen cyanide and arsenic, related to the cardiovascular system, appear to be significant with just one cigarette per day.

\section{DISCUSSION}

This paper presents a list of 158 known chemicals in cigarette smoke, along with yields and estimates of cancer and noncancer risk indices. It is recognised that these indices are not directly related to the risks the smoker experiences, since there is not a direct correlation between yields measured by the ISO method and actual human exposure. ${ }^{22}$ The methodology employed in this framework is based on that used to estimate cancer risks to communities living near chemical facilities in California and other States. However, it should be noted that this method has not been validated for cigarette smoke, and risk assessment generally is rarely capable of being validated through long term epidemiology studies. Nevertheless, we believe that the basic concept of hazard being a product of concentration and potency would apply to cigarette smoke as with any pollution source.

The list should be viewed as a subset of all possible chemicals found in the complex mixture of cigarette smoke; however, a large majority of chemicals with toxicological data are thought to be included. The current analysis is intended to provide a basis for discussion of the relative toxicological hazards posed by individual chemical constituents for cigarette smokers.

The summed crude estimates for cancer risk indices suggest risk levels that are about fivefold below those derived from the observed cancer incidence attributable to smoking in the USA. A possible explanation for this discrepancy includes the use of ISO yield data as opposed to more realistic estimates of actual delivery (That is, "intense" smoking yields). Studies have found that this difference can span several-fold. ${ }^{3}$ Another source of underestimation of carcinogenicity is the lack of cancer potency factors for all possible human carcinogens (that is, some of the nitrosamines lack potency values). We have also assumed for the purposes of this study that the potency for NNK, a well known tobacco specific nitrosamine, is equivalent to that of another tobacco specific nitrosamine, NNN. If NNK is much more potent than NNN, we will have biased our risk estimates downward. We also did not quantitatively factor into the CRI the contribution made by free radicals, which can cause DNA damage. Lastly, it is likely that cigarette smoke, containing a number of tumour initiating and tumour promoting agents may provide a favourable environment for tumour promotion (e.g. synergistic interactions), thereby increasing the overall cancer potency from what would be estimated from the sum of all components treated separately. The combination of these factors provides a plausible explanation for the difference between risk estimates and observed rates of cancer. Therefore, this analysis shows that the risk assessment approach has value for assessing the relative contributions to cancer risk, but obvious limitations in estimating the absolute magnitude of that risk.

While we attempted to use a representative yield rating by selecting a midpoint of a range of values, it is possible that this results in an under- or overestimation of the true delivery. Ideally, sales weighted yields from various brands would be used, and "intense" smoking conditions compared with standard ISO conditions. This type of information was not available at the time of this review, but could be done in future analyses.

The vast mixture of different chemicals in cigarette smoke can affect almost every organ system in the body, given sufficient duration of exposure. This is clearly illustrated by the considerable cancer and non-cancer risk values obtained even from exposure to a single cigarette per day.

The cancer and non-cancer risk indices presented are not intended to be benchmarks of absolute risk, but rather to be illustrative of the relation between the different smoke constituents and chemical classes in terms of priorities for cancer and non-cancer risk. A much more exhaustive examination of these issues would be necessary to reach definitive conclusions about the true contribution of each chemical component. This would need to include close scrutiny of the degree of confidence in each of the CPF and REL values, as well as confirmations using epidemiological evidence utilising the best approximations of real delivered doses. The limitations of this approach have been previously recognised..$^{23}$ Despite the limitations and assumptions surrounding the use of CPFs, we believe they are a useful tool for such an analysis. The use of CPF values provides risk estimates that are of the same order of magnitude to the observed mortality statistics, and it is suggested that these values, though largely derived from animal studies, provide a reasonable basis for toxic constituent prioritisation for carcinogens.

Considering the uncertainties involved in summing the results of laboratory animal studies together for such a complex mixture (45 carcinogens with 40 cancer potency factors), the two analyses presented are in relatively close agreement, lending support for the risk index methods involved. As already indicated, the risks in this review are likely underestimates as they do not represent real exposures during smoking, but only the yields generated under standard smoking machine conditions.

Among the constituents presenting a known or suspected cancer hazard, exposure to BDE appears to present the greatest cancer risk. However, BDE is not a group I IARC carcinogen, and it is possible that the potency factor calculated for BDE by the Cal/EPA, which is based on lung tumours in mice, assumes a relation to human cancer risk that is inappropriate. For example, recent studies indicate 
that the metabolic pathway of BDE leading to genotoxic products is considerably less prominent in humans than in rats. ${ }^{24}$ However, the CPF for BDE has recently been reviewed by $\mathrm{Cal} / \mathrm{EPA}^{25}$ with the result a lowering of the potency factor from $3.4(\mathrm{mg} / \mathrm{kg} / \mathrm{day})^{-1}$ to $0.6(\mathrm{mg} / \mathrm{kg} / \mathrm{day})^{-1}$. Therefore the $\mathrm{CPF}$ for $\mathrm{BDE}$ is based on the best current scientific assessment. On the other hand, even if it were determined that the CPF for BDE is overly conservative and should be reduced by, for example, an order of magnitude, BDE would still be a major carcinogen in cigarette smoke. The contributions of volatile organic compounds to overall cancer risk index are dominant even in the absence of BDE. Clearly, if a harm reduction effort for cigarette smoke were to be initiated, a focus should be on the reduction of volatile organic compounds. As these compounds are not normally included in the estimate of "tar", which is total particulate matter minus nicotine, the use of tar as a surrogate for carcinogenicity of cigarette smoke may be inappropriate. This would be particularly true if some modification to cigarettes increased the BDE concentration while leaving other constituents unchanged. Some important chemicals in the gaseous phase of tobacco smoke, such as benzene, are correlated with the amount of $\operatorname{tar}^{.15}$

The estimated contribution to cancer risk from nitrosamines is smaller than that for aldehydes and small organics, but is not negligible. There are four tobacco specific nitrosamines (TSNAs) that are reported in the literature: $\mathrm{N}$ nitrosoanabasine (NAB), N-nitrosoanabatine (NAT), NNK, and $\mathrm{NNN}^{26}$ Of these, NNK and NNN appear to have the greatest mutagenic potential. NNK and NNN have been shown to cause DNA adducts associated with tumours in rodents and are classified as probable human carcinogens by IARC. ${ }^{12}{ }^{27}$ Insufficient data currently exist to classify NAB and NAT with respect to human carcinogenicity and these two compounds are therefore not quantitatively factored into the hazard ranking approach. However, regulatory agencies, including the USFDA and USEPA, consider nitrosamines of any kind to be potential mutagens and cancer hazards just by virtue of their chemical structure (http://www.fda.gov/cvm/ fda/TOCs/G3pt3g.html). Nitrosamine formation is promoted by high levels of nitrate and nitrite. Tobacco nitrate levels have been reported to be correlated with the formation of several volatile nitrosamines, in addition to NAB and NAT, whereas the concentrations of NNK and NNN do not seem to be affected. ${ }^{28}$ This shows that the level of nitrosamines in cigarette smoke is a function of both existing levels of some types of TSNAs in tobacco (that is, NNK and NNN), and those nitrosamines that are products of chemical reactions during combustion in the presence of nitrate (NAB and NAT).

Volatile products of combustion also figure prominently in the potential risk to the cardiovascular system, with carbon monoxide and hydrogen cyanide as highly significant contributors to the NCRI.

Major target organ systems in which non-cancer effects of smoking occur include the respiratory system, cardiovascular system, reproductive system, the eyes, and the nervous system. The current analysis shows that even a single cigarette per day provides exposures to a smoker that would be expected to exceed hazard indices for respiratory and cardiovascular effects. Fetal development, including birth weight, can also be affected but was not specifically examined in this analysis.

The respiratory irritation NCRI of cigarette smoke appears to be driven by acrolein, acetaldehyde, and formaldehyde. A substantial amount of human exposure data have been factored into the RELs for these compounds, and so the degree of uncertainty about the RELs is minimal. It is implicit, but not firmly established, that exposure to atmo- spheres that exceed the RELs for chronic respiratory irritation and inflammation has a direct relation to chronic respiratory disease (asthma, or other chronic lung diseases, including emphysema).

The NCRI estimates for cardiovascular toxicity rely more heavily upon extrapolations from primate and other lab animal data, and the compounds involved have a variety of toxicological mechanisms that may not be additive. Therefore these calculations involve a greater degree of uncertainty. However, regardless of the issue of additivity of mechanisms, hydrogen cyanide and arsenic exposures alone after only a single cigarette over a chronic period are expected to result in potential cardiovascular toxicity. The NCRI approach unfortunately does not allow for an estimation to be made of the number of individuals likely to develop heart disease. It should be emphasised that considerably greater amount of information exists on dose-response relations of carcinogens and respiratory toxicants than with cardiovascular toxicants, and this is related to the degree of confidence one has in the three types of hazard ranking.

That cigarette smoking causes respiratory and cardiovascular disease requires no further proof, and while this analysis may initially seem as though it is proving the obvious, it is instructive to observe the relative breakdown of contributions to these known adverse health effects on a chemical by chemical basis.

Estimates of yield for intense or compensatory smoking are often $2-3$ fold higher than the values for normal smoking. ${ }^{29}$ This fact could contribute to the underestimate of observed cancer rates using the current analysis. While factoring this increased exposure into the risk estimates would increase the estimated risks and arguably be more representative of the real exposures and risks, the relative contribution to risk from each chemical constituent is unlikely to be changed.

Although benzo(a)pyrene and other carcinogenic PAHs are widely cited as important carcinogens in cigarette smoke, this analysis surprisingly showed that the potential risks from these compounds were low in comparison to many other compounds in smoke. Even if the CRIs from all the PAHs suspected to be human carcinogens were combined in mainstream smoke, the risk index would give a ranking that is still lower than the 16th highest ranked compound, NDEA. Therefore it appears that PAH's may be receiving a greater level of attention than they actually deserve in relation to the other chemical constituents in tobacco smoke.

In conclusion, the toxicological risk index assessment framework provides a scientific basis for ranking chemical hazards in cigarette smoke, and prioritising these compounds in terms of their contribution to toxicity of cigarette smoke. While we find this approach useful for ranking individual chemical compounds within the smokestream, it should be noted that a reduction in one or even all of these components does not necessarily equate to a reduction in risk, since this would depend on how the cigarette was smoked. While pursuit of a "harm reduction" aspect to tobacco control policy is controversial, it is vital that any measures taken to target and reduce specific compounds in cigarette smoke be based on objective, scientific analyses. We would encourage the use of these methods in ranking hazardous compounds in cigarette smoke, and to encourage the further development of RELs and CPFs for many of the remaining chemicals in cigarette smoke that have gone thus far uncharacterised in terms of their quantitative contributions to illnesses from smoking.

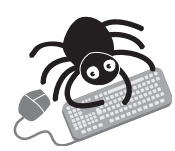

To view table 1 visit the Tobacco Control websitehttp://www.tobaccocontrol.com/supplemental 


\section{What this paper adds}

It has been known for some time that cigarette smoke contains a vastly diverse mixture of chemicals that have carcinogenic and other toxic properties. However, there has been no systematic analysis of these compounds with an aim to condense them into one risk assessment framework to prioritise harmful chemical constituents.

This paper provides a risk assessment framework for cigarette smoke constituents. We have grouped together all of the reported known or suspected carcinogens and other toxicants with published potency values in order to prioritise those that contribute most to cancer, as well as cardiovascular and respiratory disease.

\section{Authors' affiliations}

J Fowles, Institute of Environmental Science and Research, Ltd, Porirua, New Zealand

E Dybing, Division of Environmental Medicine, Norwegian Institute of Public Health. Oslo, Norway

\section{REFERENCES}

1 World Health Organisation Tobacco Free Initiative. Oslo, Norway: WHO Workshop on Advancing Knowledge on Regulating Tobacco Products, February 2000

2 Borgerding MF, Bodnar JA, Chung HL, et al. Chemical and biological studies of a new cigarette that primarily heats tobacco. Part 1. Chemical composition of mainstream smoke. Food and Chemical Toxicology 1998;36:169-82.

3 British Columbia Ministry of Health. 2000. http://www.cctc.ca.

4 Dybing E, Soderlund EJ, Gordon WP, et al. Studies on the mechanism of acetamide hepatocarcinogenicity. Pharmacol Toxicol 1987;60:9-16.

5 California Environmental Protection Agency. Cancer Potency Factors for Propsition 65 Carcinogens. Office of Environmental Health Hazard Assessment. 2001. www.oehha.ca.gov.

6 U.S. Environmental Protection Agency. Integrated Risk Information System(IRIS) database. www.epa.gov/iris.

7 Menzie Cura Associates, Inc. Estimating risk to cigarette smokers from smoke constituents in proposed "testing and reporting of constituents of cigarette smoke" regulations. August 1999. Prepared for: Massachusettes Tobacco Control Program, Boston, Massachusetts, 1999.

8 Fowles J, Bates M. The toxic constituents of tobacco and tobacco smoke: priorities for harm reduction. A report to the New Zealand Ministry of Health. March 2000, www.ndp.govt.nz.

9 World Health Organization. Joint Expert committee on Food Additives and Contaminants (JECFA). Safety Evaluation of Certain Food Additives and Contaminants: Polychlorinated dibenzodioxins, polychlorinated bibenzofurans, and coplanar polychlorinated biphenyls. Food Additive Series, 2002:48.

10 Lofroth G, Zebuhr Y. Polychlorinated dibenzo-p-dioxins (PCDDs) and dibenzofurans (PCDFs) in mainstream and sidestream cigarette smoke. Bulletin of Environmental Contamination and Toxicology 1992;48:789-94.
11 California Environmental Protection Agency. Risk assessment guidelines: chronic reference exposure levels for airborne toxicants. Office of Environmental Health Hazard Assessment. 2001. www.oehha.ca.gov.

12 International Agency for Research on Cancer (IARC). Monographs on the carcinogenicity of chemical substances. 1999. 193.51.164.11/monoeval/ grlist.html.

13 U.S Department of Health and Human Services. NTP. Eighth Report on Carcinogens: Summary report. North Carolina: Research Triangle Park, 1998.

14 International Agency for Research on Cancer (IARC). Monographs on the carcinogenicity of chemical substances. 1986:38, 193.51.164.11/ monoeval/grlist.html

15 Smith CJ, Livingston SD, Doolittle DJ. An international literature survey of "IARC Group I carcinogens" reported in mainstream cigarette smoke. Food and Chemical Toxicology 1997;35:1107-30.

16 Agency for Toxic Substances and Disease Registry (ATSDR). Toxicological profile for hydrogen sulfide. July 1999. http://www.atsdr.cdc.gov/ toxprofiles/tpl 14.html.

17 Daisey JM, Mahanama KRR, Hodgson AT. Toxic volatile organic compounds in simulated environmental tobacco smoke: emission factors for exposure assessment. Journal of Experimental and Analytical Environmental Epidemiology 1994;8:313-34.

18 California Environmental Protection Agency. Air Resources Board. National ambient air criteria for carbon monoxide. 2001. www.arb.ca.gov/aqs/ aaqs2.pdf

19 US Department of Health and Human Services. Reducing the health consequences of smoking: 25 years of progress. A report of the Surgeon General, 1989. Rockville, Maryland: Public Health Service, Centers for Disease Control, Office on Smoking and Health, 1989. (DHHS Publication No (CDC) 89-8411.) (table 7).

20 Agency for Toxic Substances and Disease Registry (ATSDR). Toxicological profile for vinyl acetate. www.atsdr.cdc.gov/toxprofiles/TP59-c5.pdf. Accessed January 2003

21 Shopland D. Tobacco use and its contribution to early cancer mortality with a special emphasis on cigarette smoking. Environmental Health Perspectives 1995; 103(suppl 8):131-41.

22 Benowitz N, Hall SM, Herning Rl, et al. Smokers of low yield cigarettes do not consume less nicotine. N Engl J Med 1983;309:139-42.

23 Rustemeier K, Stabbert $\mathrm{R}$, Haussmann $\mathrm{HJ}$, et al. Evaluation of the potential effects of ingredients added to cigarettes. Part 2: chemical composition of mainstream smoke. Food and Chemical Toxicology 2002:40:93-104.

24 Van Sittert NJ, Megens HJJ, Watson WP, Biomarkers of exposure to 1,3butadiene as a basis for cancer risk assessment. Toxicological Science 2000;56:189-202.

25 California Environmental Protection Agency. 2002. Correction to the cancer potency factor for 1,3-butadiene. http://www.oehha.org/pdf/ corrections.pdf. Accessed December 2002.

26 Mitacek EJ, Brunnemann KD, Hoffmann D, et al. Volatile nitrosamines and tobacco-specific nitrosamines in the smoke of Thai cigarettes: a risk factor for lung cancer and a suspected risk factor for liver cancer in Thailand. Carcinogenesis 1999;20:133-7.

27 Hecht S. DNA adduct formation from tobacco-specific $\mathrm{N}$-nitrosamines Mutation Research 1999:424:127-42.

28 Fischer S, Spiegelhalder B, Eisenbarth J, et al. Investigations on the origin of tobacco-specific nitrosamines in mainstream smoke of cigarettes. Carcinogenesis 1990;11:723-30.

29 Physicians for a Smokefree Canada. 2000. http://www.smoke-free.ca/ eng_issues/etsoutsmokers.htm.

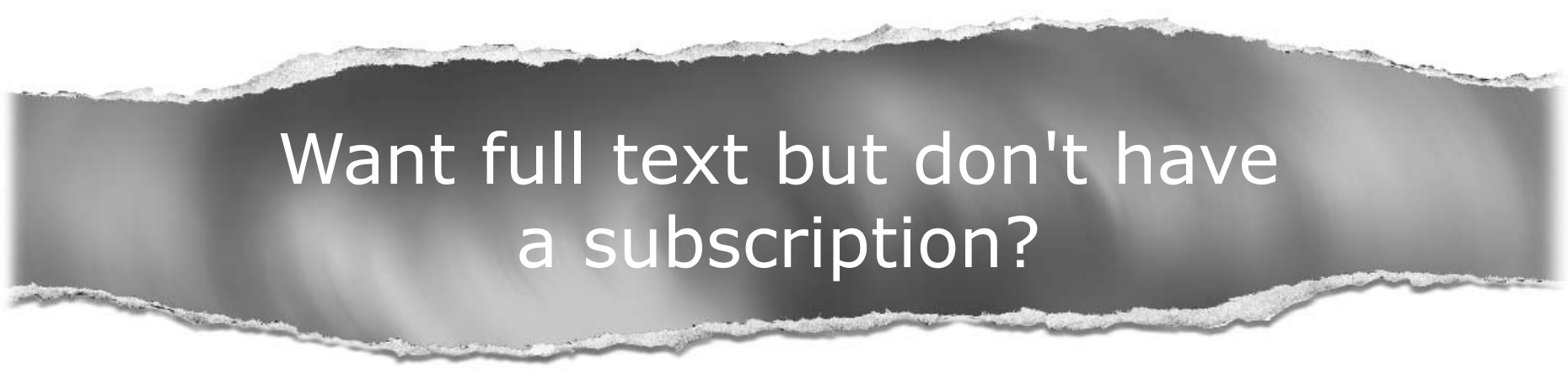

\section{Pay per view}

For just $\$ 8$ you can purchase the full text of individual articles using our secure online ordering service. You will have access to the full text of the relevant article for 48 hours during which time you may

download and print the pdf file for personal use. 